\title{
Proton Therapy Review: Proton Therapy from a Medical
}

\author{
Se Byeong Lee ${ }^{(1)}$ \\ Department of Radiation Oncology \& Proton Therapy Center, National Cancer Center, Goyang, Korea
}

Received 27 July 2020

Revised 6 September 2020

Accepted 9 September 2020

Corresponding author

Se Byeong Lee

(sblee@ncc.re.kr)

Tel: 82-31-920-1729

Fax: 82-31-920-0149
With hope and concern, the first Korean proton therapy facility was introduced to the National Cancer Center (NCC) in 2007. It added a new chapter to the history of Korean radiation therapy. There have been challenging clinical trials using proton beam therapy, which has seen many impressive results in cancer treatment. Compared to the rapidly increasing number of proton therapy facilities in the world, only one more proton therapy center has been added since 2007 in Korea. The Samsung Medical Center installed a proton therapy facility in 2015. Most radiation oncology practitioners would agree that the physical properties of the proton beam provide a clear advantage in radiation treatment. But the expensive cost of proton therapy facilities is still one of the main reasons that hospitals are reluctant to introduce them in Korea. I herein introduce the history of proton therapy and the cutting edge technology used in proton therapy. In addition, I will cover the role of a medical physicist in proton therapy and the future prospects of proton therapy, based on personal experience in participating in proton therapy programs from the beginning at the NCC.

Keywords: Proton therapy, Passive scattering, Pencil beam scanning, Flash therapy, Acr therapy

\section{Introduction}

Proton therapy (PT) was introduced at the National Cancer Center (NCC) in 2007. Compared to other new modalities in radiation therapy, the number of PT sites has not increased in the last 13 years in Korea, except for at the Samsung Medical Center (SMC). SMC opened the second PT center in 2015. Due to the expensive price of PT facilities, even major Korean hospitals are reluctant to install them. Particle therapy has the most benefit compared with other beam modalities in radiation therapy; it can deliver the maximum dose to the tumor target and minimize exposure dose to organs at risk (OARs). It comes from the Braggpeak characteristics of accelerated ion particles [1]. There are two kinds of particles, proton and carbon ions, conventionally used in clinics. The Bragg-peak of the proton beam can be described using the Bethe-Bloch formula in Eq. (1), which calculates kinetic energy transfer of charged particles by electromagnetic interactions in matter.

$-\frac{d E}{d x}=2 \pi \mathrm{N}_{\mathrm{a}} \mathrm{r}_{\mathrm{e}} \mathrm{m}_{\mathrm{e}} \mathrm{c}^{2} \rho \frac{Z}{A} \frac{\mathrm{z}^{2}}{\beta^{2}}\left[\ln \left(\frac{2 \mathrm{me} \gamma 2 \mathrm{v} 2 \mathrm{~W}_{\max }}{\mathrm{I}^{2}}\right)-2 \beta^{2}-\delta-2 \frac{\mathrm{C}}{\mathrm{Z}}\right](1)$

Where $2 \pi \mathrm{N}_{\mathrm{a}} \mathrm{r}_{\mathrm{e}}{ }^{2} \mathrm{~m}_{\mathrm{e}} \mathrm{c}^{2}=0.1535 \mathrm{MeVcm}^{2} / \mathrm{g}, \mathrm{r}_{\mathrm{e}}$ : classical electron radius $=2.817 \times 10^{-13} \mathrm{~cm}, \mathrm{~m}_{\mathrm{e}}$ : electron mass, $\mathrm{N}_{\mathrm{a}}$ : Avogadro's number $=6.022 \times 10^{23} / \mathrm{mol}$, I: mean excitation potential, $\mathrm{Z}$ : atomic number of absorbing material, A: atomic weight of absorbing material, $\rho$ : density of absorbing material, $z$ : charge of incident particle in units of $e, \beta=v / c$ of incident particle, $\gamma=1 / \sqrt{1-\beta^{2}}, \delta$ : density correction, C: shell correction, $\mathrm{W}_{\max }$ : maximum energy transfer in a single collision.

Actually, the linear energy transfer (LET) of ion particles is proportional to the square of the number of the charge, LET $\sim \mathrm{Z}^{2}$. Protons are the lightest atom of $\mathrm{Z}=1$ and are one of major elements in the formation of the human body. Also, 
they do not make any fragments and give no exit dose over the beam range. Heavy ion particles of more than 10 atomic number give too high LET on the normal organs in the human body. So, only helium, carbon, nitrogen and oxygen are adaptable to clinics. At present, over $80 \%$ of particle therapy facilities use proton beams for patient treatment. An LET of around $100 \mathrm{keV} / \mu \mathrm{m}$ can give a maximum relative biological effectiveness (RBE). The LET of a proton beam [2] is small; $\sim 20 \mathrm{keV} / \mu \mathrm{m}$ in the human body. It gives around 1.1 $\mathrm{RBE}$ and is recommended for clinic use $[3,4]$.

The purpose of this review paper is to introduce the history of PT and the cutting edge technology in PT. In addition, I will describe the role of a medical physicist in PT and the future prospects of PT, based on personal experience in participating in PT programs from the beginning in NCC.

\section{History of PT}

\section{Proton, accelerator, and proton treatment}

Ernest Rutherford found a light particle in an alpha particle and nitrogen gas collision experiment in the early 1900s. He named it "proton," coming from "protos," meaning "first." In 1929, Ernest Orlando Lawrence invented a cyclotron to accelerate protons and developed it further during the 1930s to accelerate protons for a high kinetic energy [5]. The idea to use proton beams for cancer treatment was proposed by Dr. Robert Rathburn Wilson in 1946. The preclinical research to understand the biological effects of the proton beam was done by Cornelius Anthony
Tobias and John Hundale Lawrence, M.D., younger brother of Ernest O. Lawrence, using a 184-inch synchrocyclotron at the Lawrence Berkeley Laboratory in the 1940s to 1950s. The first PT patient in the world was a metastatic breast cancer case who received beam irradiation in the pituitary gland region. John H. Lawrence treated the patient at the University of California, Berkeley, in 1954. In 1957, Uppsala University in Sweden started providing PT. In 1961, the Massachusetts General Hospital in the United States started PT using a cyclotron from Harvard. Before the 1990s, more than 10 facilities had treated patients using proton beams at the laboratory level [6].

The first hospital-based PT facility was installed in Loma Linda University Hospital in 1990. Actually, it was the first facility designed for commercial purposes, and then the number of PT facilities in the world increased rapidly. From 1954 to 2018, over 190,000 patients got PT at 78 facilities worldwide (Fig. 1) [7].

\section{History of PT in Korea}

The first PT facility project in Korea was suggested by the government in the early 2000s. The NCC carried it forward with a government budget in 2001 through a contract with Ion Beam Application (IBA). The facility consists of a cyclotron that can accelerate proton beams to $230 \mathrm{MeV}, 2$ gantry treatment rooms, and 1 fixed beam treatment room. IBA started the installation of the PT system at the beginning of 2005 and finished at the end of 2006. The passive beam irradiation modes were installed for patient treatment with a

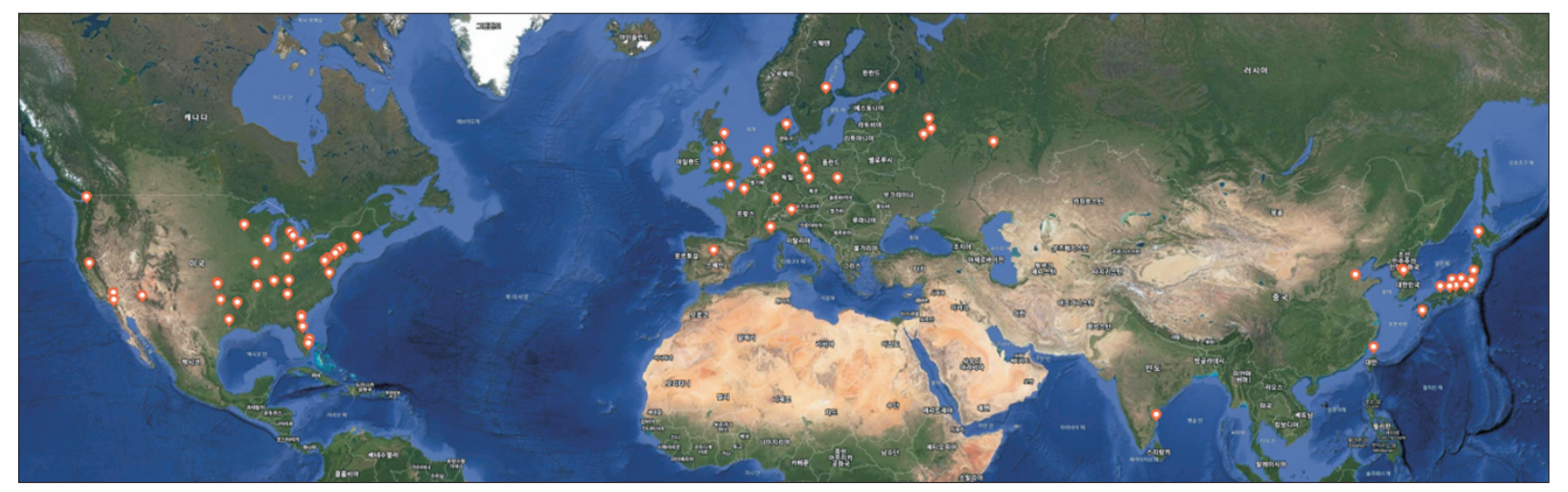

Fig. 1. Proton therapy facilities in operation worldwide [7]. 


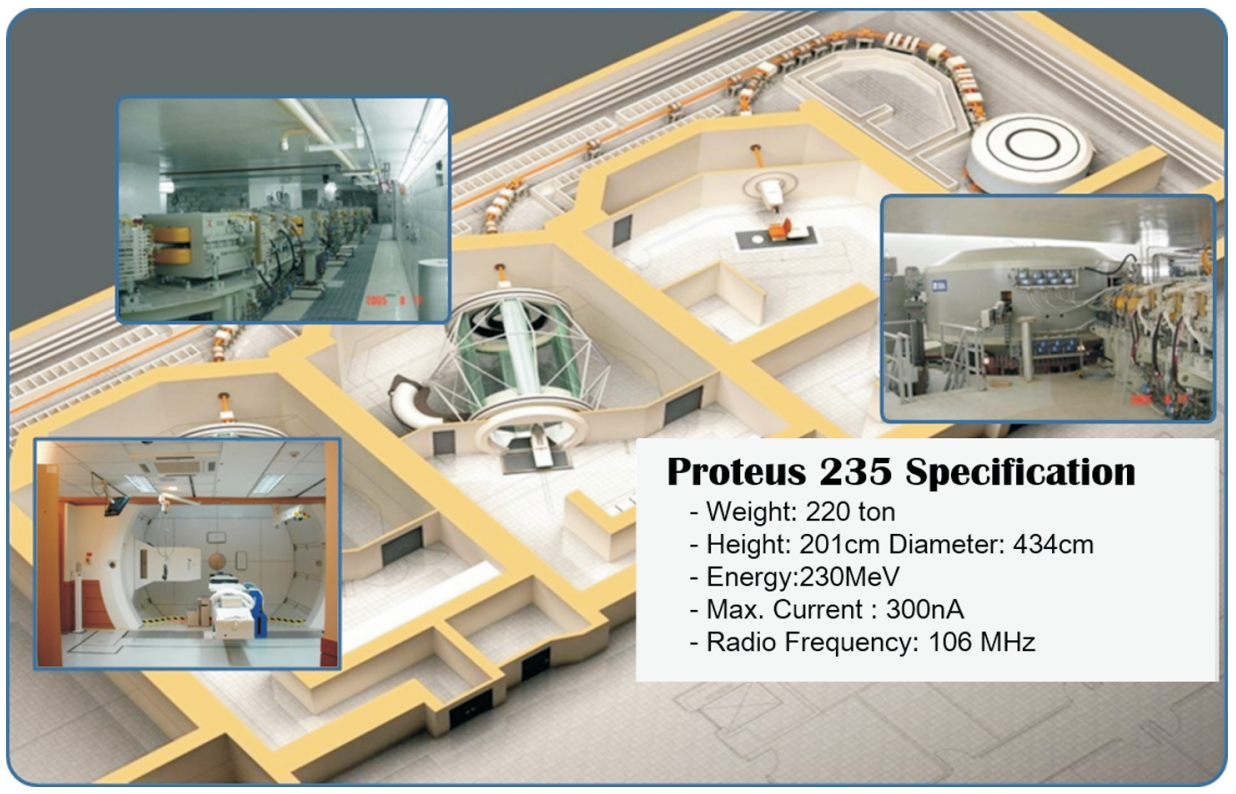

Fig. 2. The layout of the proton therapy facility at the National Cancer Center.

2-dimensional (2D) based patient positioning system and a 6 degree of freedom couch system at the initial installation stage. The wobbling beam mode and pencil beam scanning (PBS) mode were upgraded in 2010 and 2015, respectively (Fig. 2). The first PT patient was a prostate cancer case who got treatment in March 2007 [8]. By the end of 2018, a total of 2,914 patients had received proton treatment at the NCC Korea (Fig. 3).

The SMC in Seoul opened the second PT facility in Korea in 2015. They introduced a PT system manufactured by Sumitomo Heavy Industry (Tokyo, Japan). The accelerator is a cyclotron with $230 \mathrm{MeV}$ proton beam energy. It has 2 gantry treatment rooms with PBS dedicated nozzles and multipurpose nozzles. SMC PT started patient treatment at the end of 2015 and treated 1,250 patients by the end of 2018 (Fig. 4).

\section{The Cutting Edge PT System}

PT facilities are complex, with an accelerator, a beam translation system, a gantry system, a beam irradiation nozzle, a patient positioning system, and an imaging system. Unlike the medical linear accelerator system for X-ray and electron treatment, it requires an independent building designed for neutron radiation shielding and a huge space to install beam generation, delivery, irradiation devices

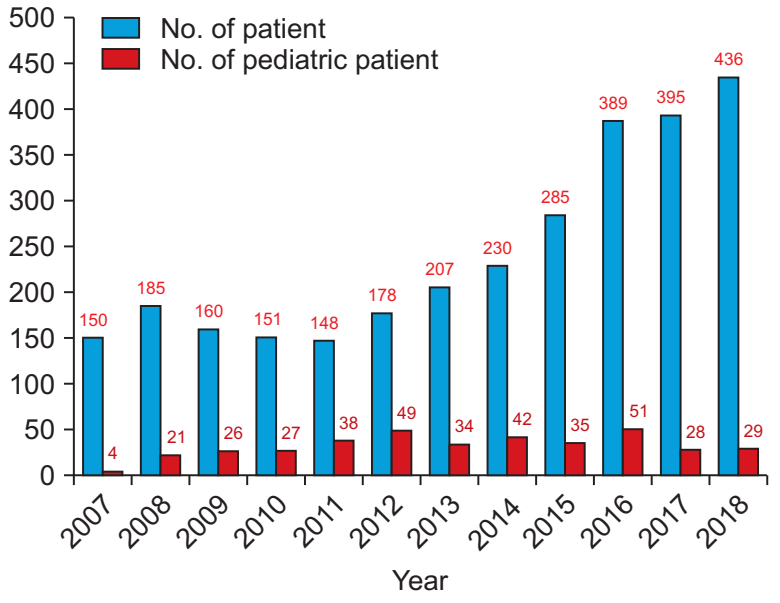

Fig. 3. Statistics of proton patients at the National Cancer Center.

and power, and a cooling plant. Here, the principal parts of proton therapy system are introduced including recent technical development. Also, the new technologies in PT are introduced.

\section{The principal parts of the PT system}

\section{1) Accelerator}

For clinical use, there are two types of accelerators, the cyclotron and synchrotron, for proton beam acceleration. They accelerate proton beam up to $230-250 \mathrm{MeV}$ kinetic energy to reach the tumor depth in patient body. Fig. 5 shows 


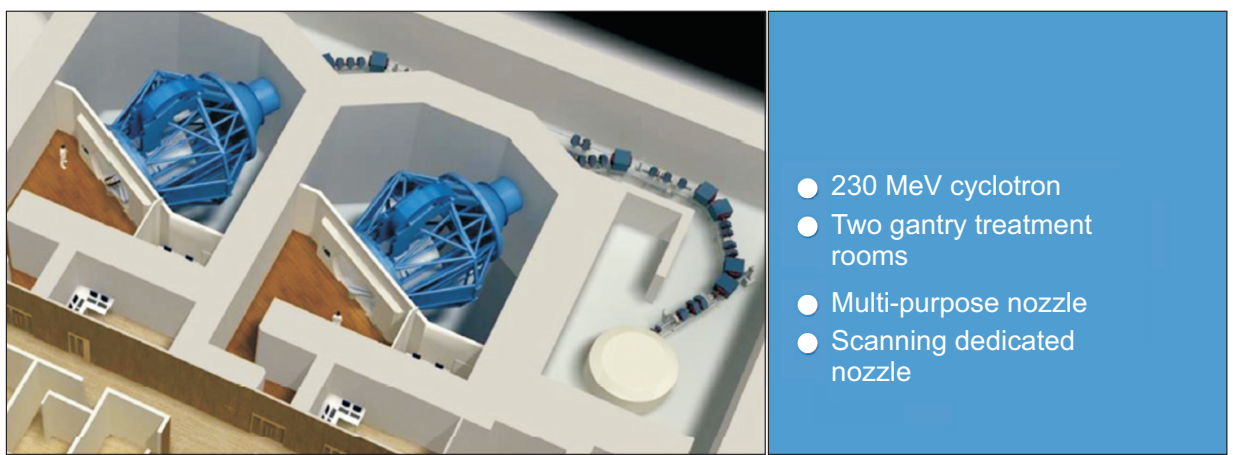

Fig. 4. The layout of the Samsung Medical Center proton facility in Seoul.

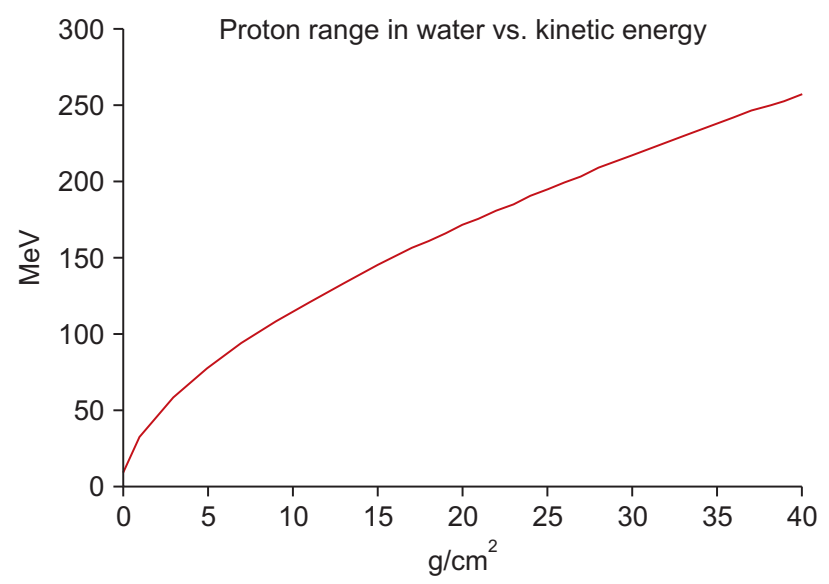

Fig. 5. The correlation between proton beam range in water and kinetic energy.

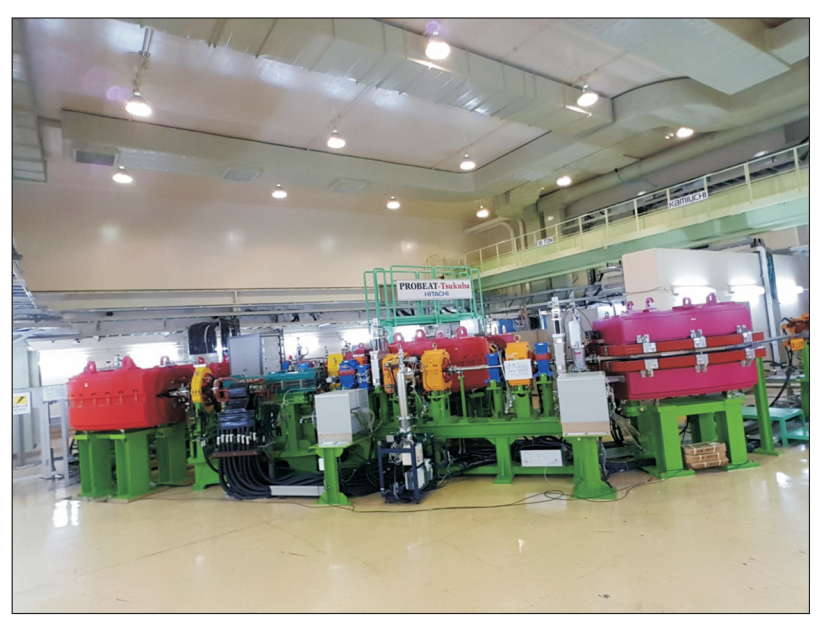

Fig. 7. Synchrotron for proton therapy at the Tsukuba University Hospital in Japan.

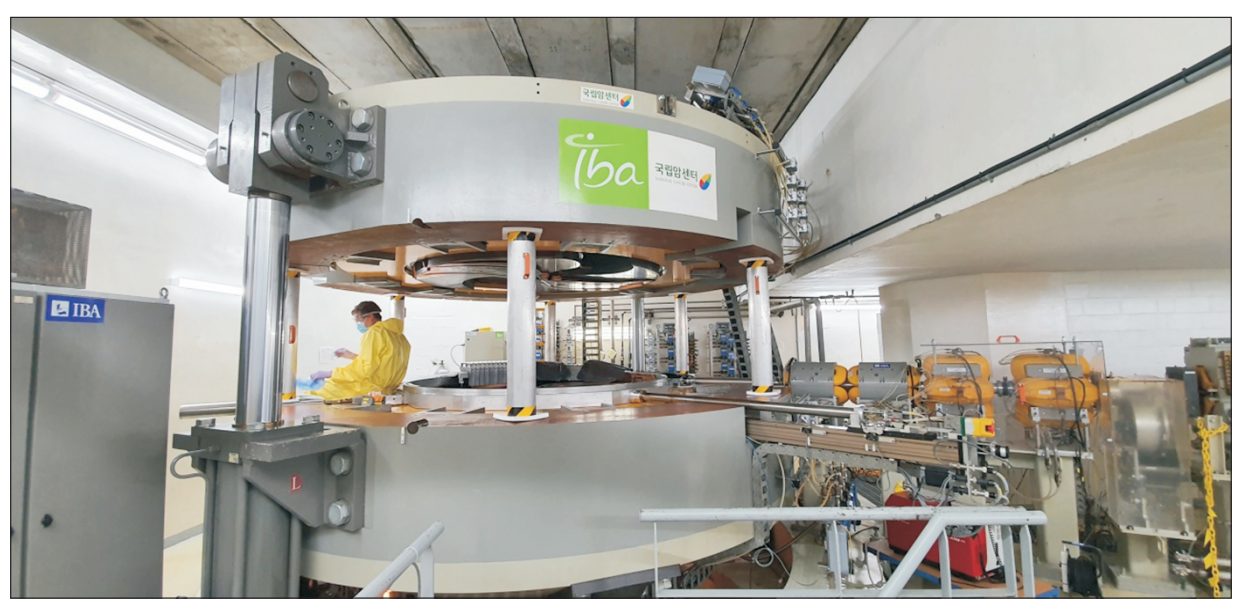

Fig. 6. Ion Beam Application (IBA, Louvain-la-Neuve, Belgium) Cyclotron for proton therapy at the National Cancer Center, Korea. the relation between proton kinetic energy and penetration depth in water.

The basic concept of an accelerator is that it accelerates a charged particle with electromagnetic force. A cyclotron has a cylindrical shape and consists of radio frequency
(RF) cavities, two hollow semicircular RF electrodes (dees), fixed magnetic field poles, and an ion source at the center. It generates a fixed beam energy, and one of its advantages is an easily adjustable beam current. There are two kinds of cyclotron: the isochronous cyclotron and the synchro- 
cyclotron. The isochronous cyclotron is the original type of cyclotron and has a fixed RF. Generally, it is 3.5 to $5 \mathrm{~m}$ in diameter, which is larger than the synchrocyclotron for PT. The synchrocyclotron has a compact size, less than $2 \mathrm{~m}$ diameter, and uses a super conduction magnet technique (Fig. 6).

A synchrotron is a closed loop type accelerator and was invented by Vladimir Veksler in 1944 [6]. The first synchrotron was installed by Edwin McMillan in 1945. It consists of bending magnets and acceleration RF parts. The beam energy can be changed easily with an adjustable magnetic field and RF on the synchrotron loop (Fig. 7). The synchrotron accelerator can adjust beam energy and reduce beam

Table 1. Comparison between the synchrocyclotron and the synchrotron

\begin{tabular}{lll}
\hline \multicolumn{1}{c}{ Item } & \multicolumn{1}{c}{ (Synchro-)Cyclotron } & \multicolumn{1}{c}{ Synchrotron } \\
\hline Time structure & Continuous (SC: pulsed) & Beam spills \\
Fast energy change & Possible-energy degrader & Difficult-next beam spill \\
Radiation shielding & Hot point at energy degrader & General \\
Beam intensity & High (SC: low) & Limited (per spill) \\
Beam intensity stability (\%) & $3-5$ & $15-20$ \\
Radius of accelerator (m) & $3.5-5.0($ SC: $<2.0)$ & $6-8$ \\
Scattering/spot scanning & O.K. & O.K. \\
Fast continuous scanning & O.K. (SC: no) & Difficult \\
\hline
\end{tabular}

SC, Syncho-Cyclotron.

a Scattering

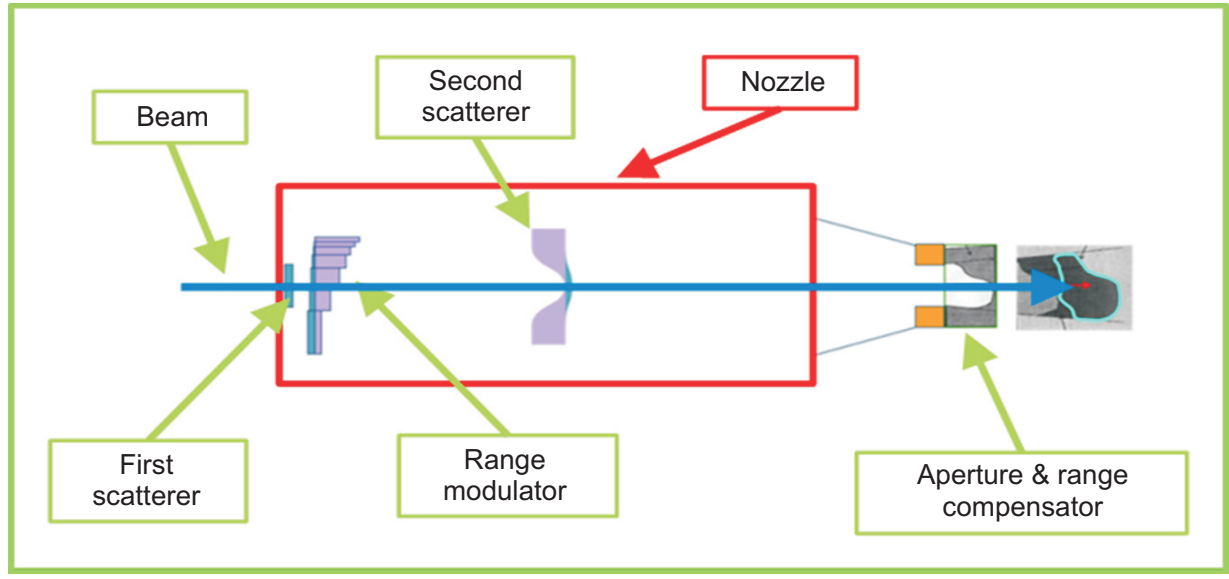

b Scanning

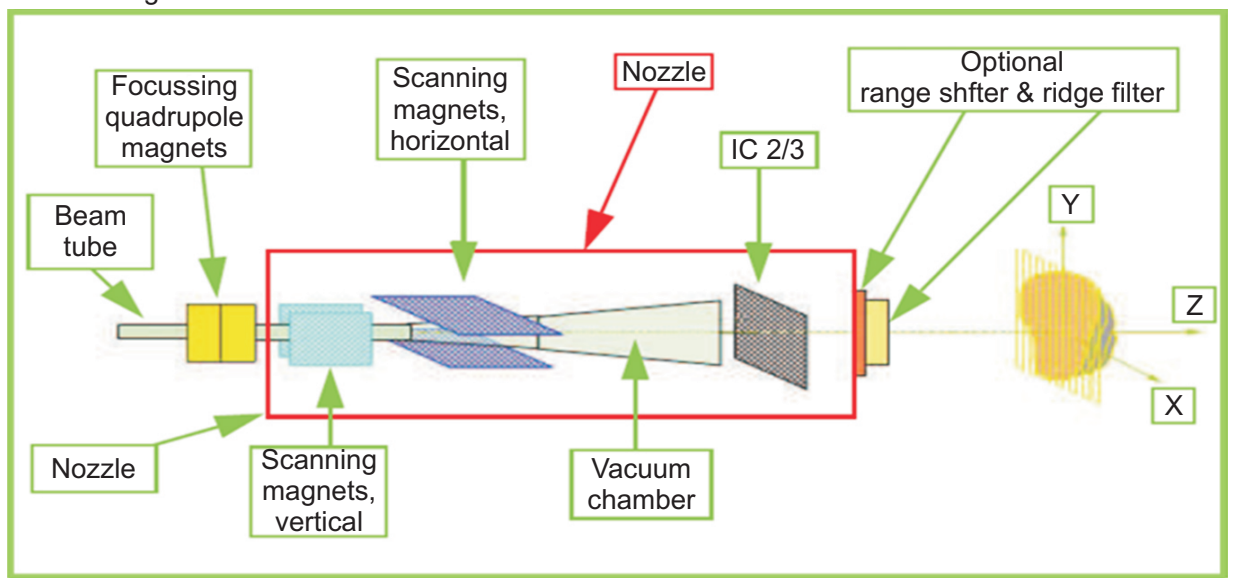

Fig. 8. The proton beam nozzle for (a) scattering mode and (b) pencil beam scanning. 
loss in the acceleration process. However, it has more complicated components and a pulsed beam structure compared with the cyclotron. Table 1 shows the specification comparison of both types of accelerators.

There have been several trials to develop a new accelerator type with a more compact and simple design, like the fixed-field alternating gradient accelerator (FFAG), the laser plasma accelerator, the dielectric wall accelerator, and so forth. They are still under research and development, but it is still unclear whether they are a practical PT solution. To cover the whole body region, the accelerator should accelerate proton beam kinetic energy up to 230 to $250 \mathrm{MeV}$ to reach more than $32 \mathrm{~g} / \mathrm{cm}^{2}$ depth in the patient's body. Also, it should provide a stable and adequate beam current to make the treatment beam comparable with conventional Xray treatment.

\section{2) Beam nozzle system}

The role of beam nozzle system is to make a field shape and dose painting for patient treatment. It is located at the end of beam line. The traditional nozzle technique is a passive beam mode using a scatter and beam modulation component (Fig. 8). It requires a dedicated patient aperture and a range compensator. The uniform dose of total target volume is painted in a very short time and very stably. The width of the distal falloff is increased by the interactions with the components on the beam pathway in the nozzle. It can give a scattered secondary dose level [9] similar to X-ray intensity modulated radiation therapy (IMRT) in a patient. Particle/PBS can give a more conformal dose distribution to the target and less secondary beam distribution than pas- sive scattering. However, it is more difficult to distribute a uniform dose, and to perform sequential dose delivery, on the target with the PBS technique.

Currently, most PT vendors provide the scattering and wobbling modes optionally and PBS as default (Table 2). The PBS nozzle has a simple structure and can deliver more a precise beam but a worse dose uniformity compared to the passive beam nozzle.

\section{3) Gantry}

The particle therapy system needs a larger gantry structure compared to the X-ray linear accelerator system because it requires support for strong magnet components to bend heavy particles. Most of the PT gantry system is designed for 360 degree rotation. Nevertheless, some oneroom solutions provide limited rotational angles, such as the Mevion with 190 degrees and the IBA Proteus with 220 degrees (Fig. 9).

The recent trend is to make a compact gantry for a small footprint in the hospital, and vendors are trying to use a new magnet design with transverse alignment to the gantry rotational axis or a limited rotation angle design.

\section{4) Patient positioning system}

To achieve an exact and precise beam targeting, it is essential to have 6 degrees of freedom (DOF) for the patient couch system. There are two types of couch systems available in the proton treatment room. The conventional type has a sliding design, to the $\mathrm{x}, \mathrm{y}$, and $\mathrm{z}$ axis, and a rotational design to rotate, pitch, and roll. It generally provides stable movement and a rapid setup time compared to the robotic

Table 2. Specifications of the IBA universal nozzle, used at the NCC in Korea

\begin{tabular}{lllcc}
\multicolumn{1}{c}{ Item } & \multicolumn{1}{c}{$\begin{array}{c}\text { Double } \\
\text { scattering }\end{array}$} & $\begin{array}{c}\text { Wobbling } \\
\text { scanning }\end{array}$ & $\begin{array}{c}\text { PBS } \\
\text { (multipurpose nozzle) }\end{array}$ & $\begin{array}{c}\text { Dedicate PBS } \\
(\text { one room PT) }\end{array}$ \\
\hline Beam range & $4.5-28.4$ & $3.4-32$ & $7.5-32$ & $4.1-32$ \\
Max. field size & 24.3 & $40 \times 30$ & $40 \times 30$ & $40 \times 30(20 \times 24)$ \\
Dose rate & Ave. 3 & Ave. 1.2 & Ave. $1.0 /$ liter & $>6.0 /$ liter \\
Lateral PB/distal FO & $0.5 / 0.23$ & $0.3 / 0.13$ & - & - \\
Spot size & & - & 0.5 -high/1.1-Low & 0.35 -high/0.77-low \\
Effective SAD & 2.2 & 2.1 & 2.1 & 2.1 \\
Accessories & Aperture, range & Aperture, range & Range shifter, & Range shifter, \\
& compensator & ridge filter & ridge filter, MLC \\
\hline
\end{tabular}

IBA, Ion Beam Application; NCC, National Cancer Center; PT, proton therapy; PB, penumbra; FO, fall-off; SAD, source to axis distance; MLC, multi-leaf collimator; -, not available. 

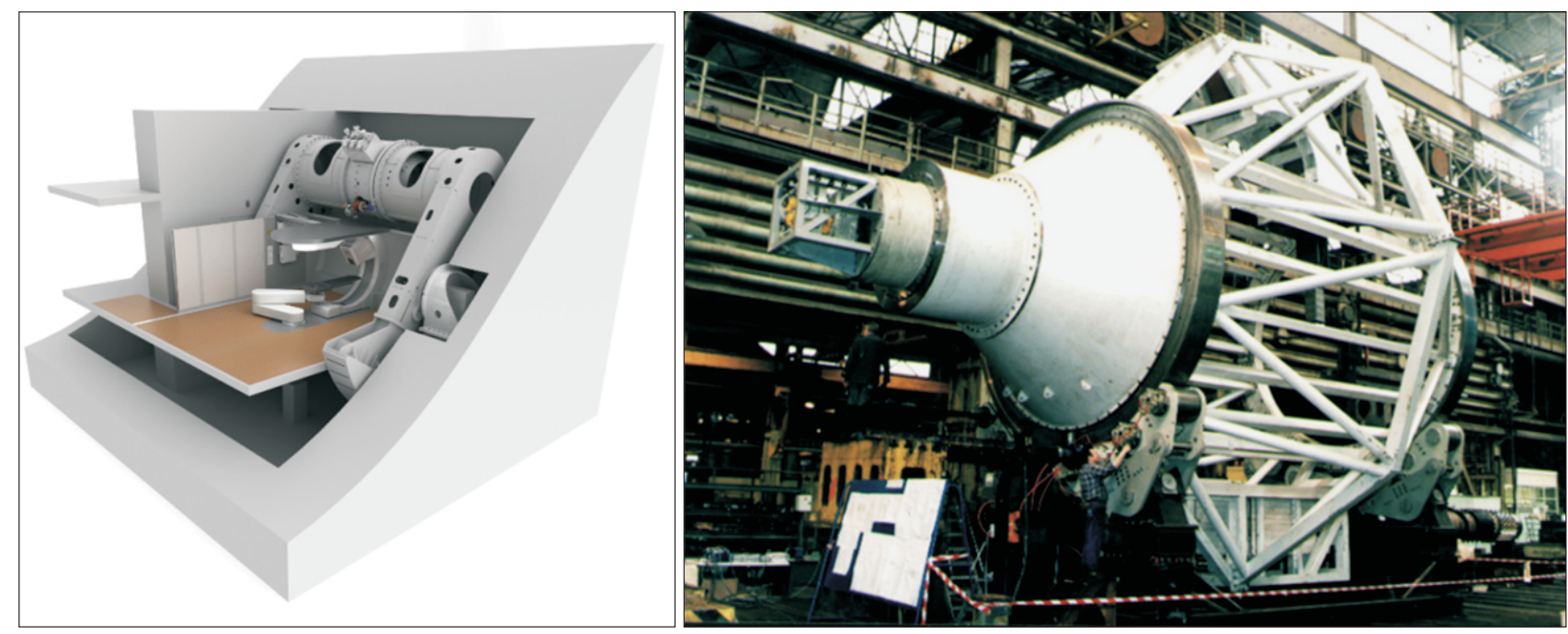

Fig. 9. The Mevion gantry (left) and the Ion Beam Application (IBA) Proteus plus gantry (right).

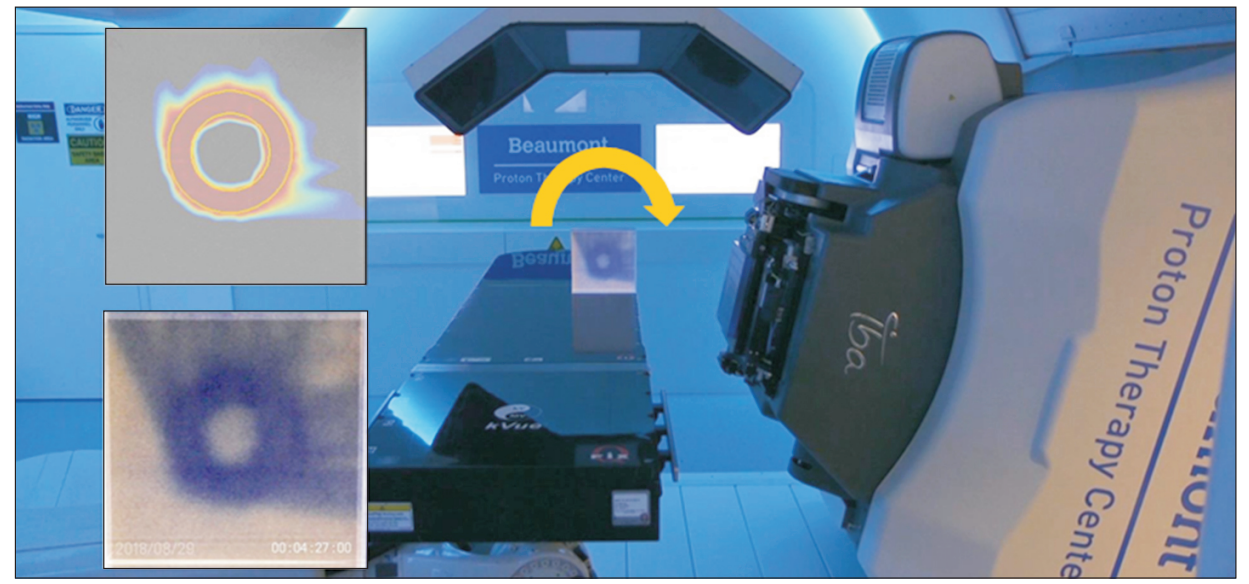

Fig. 10. Proton arc therapy demonstration on an Ion Beam Application (IBA) Proteus One, with dynamic gantry control, fast and accurate beam, an optimal treatment planning system (TPS) algorithm, and 3D dosimetry (courtesy of IBA).

couch system. The robotic couch consists of joint parts to provide $6 \mathrm{DOF}$ motion. It provides a more compact footprint and a wider patient setup region than a conventional couch system.

5) Image-guided patient alignment system

Generally, a 2D X-ray imaging flat panel system [10], fan-beam computed tomography (CT), or cone beam CT (CBCT) are used for patient alignment in the treatment room in a modern radiation therapy solution. Most of the PT solution provides 2D imaging systems as default and CT optionally.

\section{Cutting edge techniques in PT}

1) Arc therapy

The advanced beam delivery technique in radiation therapy is volumetric modulated arc therapy (VMAT). It can deliver beam treatment more precisely and more quickly to patients. Recently, the VMAT technique was introduced in PT by IBA. They developed a new control device to irradiate a fast PBS during gantry rotation (Fig. 10) [11,12].

\section{2) Flash therapy}

Flash therapy, which delivers an ultra-high dose rate of more than $40 \mathrm{~Gy} / \mathrm{s}$ and has a very short exposure time of 
less than 0.5 seconds, allows decreasing normal tissue complication probability (NTCP) and increasing tumor control probability (TCP) compared to the conventional dose rate, at least in animal models. The mechanism of flash therapy effects is still not clear. One possibility is the suppression of cytokine activation, which releases transforming growth factor-beta in normal lung tissue. Another possible mechanism is the radiation reacting with almost all of the available oxygen and liberating huge electrons, producing many more ionizations than conventional dose rates. It can maximize the differences of the reduction-oxidation reaction and free radical chemistry events between normal tissues and cancer tumors [13]. Several PT system vendors are developing a flash therapy option for PT at present [14-16].

\section{3) In vivo beam monitoring and dosimetry}

One of the big issues in PT is beam range uncertainty, because it is used in clinics [17]. There is ongoing research to measure proton treatment beam range and dose distributions in patients. One study measured the radioactive isotopes produced by particle treatment beam with a positron emission tomography (PET) detector $[18,19]$. However, there were several limitations, such as poor imaging quality, physiological washout, and organ motion issues. Other research measured prompt gamma induced by a particle treatment beam during patient treatment. From the scanning of prompt gamma distribution, the beam range can be verified (Fig. 11) [20]. Another study looked at magnetic resonance (MR) images for certain organs, which changed physiologically after particle beam exposure [21]. The preliminary studies showed that MR images of spine and liver tissues changed after proton irradiation.

\section{The Role of the Medical Physicist in PT}

Basically, the medical physicist's role in PT is similar to that for conventional radiation. The physicist must run the machine acceptance test, take beam data, commission the treatment planning system (TPS), perform regular machine quality assurance (QA) [22] and patient treatment planning \& delivery QA, and manage radiation safety. Here, these roles are reviewed.

\section{Acceptance test procedure (ATP)}

There are more than 10 PT solution providers, and each solution has been developed with its own design. So, there is a lack of user reference and guidelines for ATP. It is recommended that physicists use vendor guidelines for beam calibration and machine alignment in advance of the ATP. Actually, there are two groups of control values. One is machine parameter tolerance level values that were specified on the purchase contract. The other is machine parameter limitation values related to machine performance. Generally, the vendor engineers finish the calibration and alignment work within contractual conditions. The physicist can
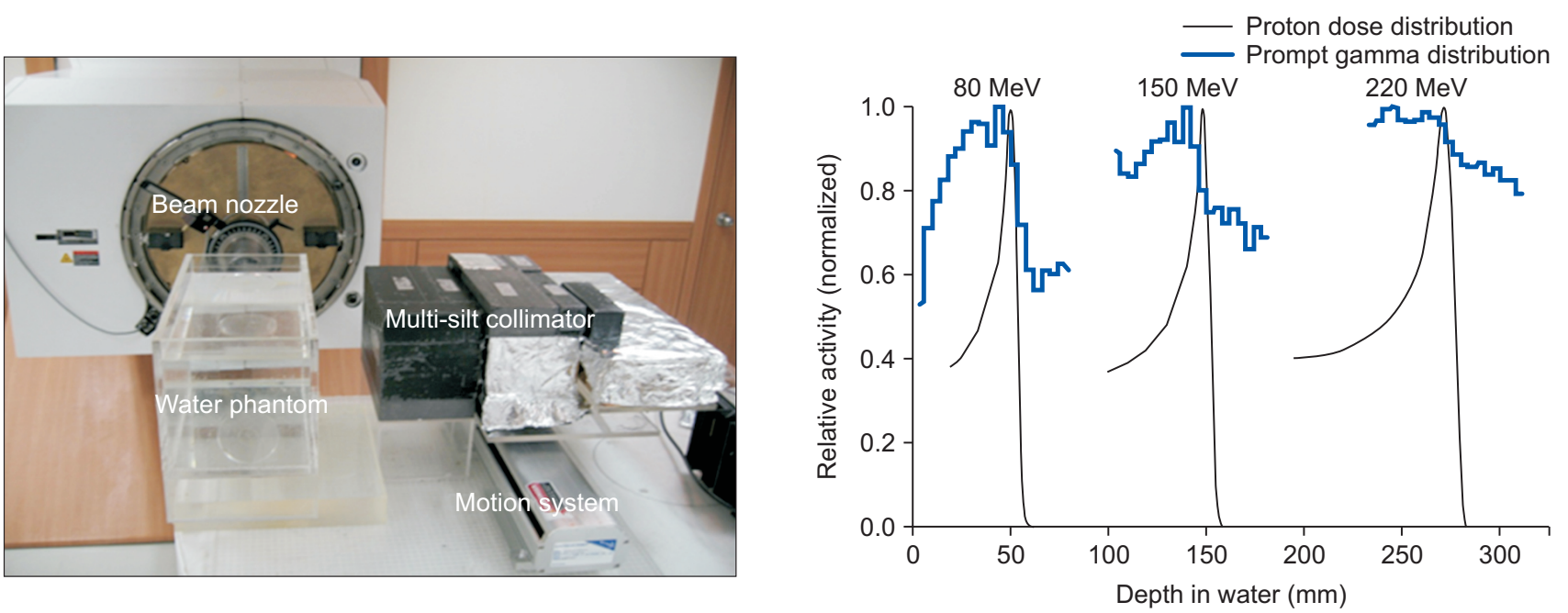

Fig. 11. A prompt gamma detector experiment with water phantom at the National Cancer Center in Korea [20]. 
cooperate with them to tune the machine more precisely over the contractual values, which can reduce the ATP time.

\section{Commission of TPS}

Commercially available TPS models for PT are limited. Varian has their own PT solution and TPS. The other PT vendors need to collaborate with TPS providers for the development of a dedicated TPS solution. Sometimes, PT customers should be involved in the development of TPS for their preferred TPS solution; however, this requires more time and cost.

For the commissioning of TPS, the physicist has two main work items: CT Hounsfield unit (HU) calibration to proton beam stopping power ratio and beam modeling [23]. The proton beam stopping power ratio values of human organs are not linear to CT HU values. So, finding the best fitting curve of CT HU versus proton stopping power for the PT system is important. It is related to beam range uncertainty in proton planning. The proton beam data measurement for beam modeling [24] requires different dosimetry tools for the X-ray and the electron beam. The current main beam delivery technique is PBS in PT. The essential beam parameters are the beam profile and irradiation depth dose distributions of the pencil beam. To measure them, the scintillator and CCD combination system [25,26] and the multilayer ionization chamber types are used. As an indirect beam measurement, the Monte Carlo simulation modeling and calculation method is useful for TPS model commissioning [27-29].

\section{PT machine $\mathrm{QA}$}

The American Association of Medical Physics (AAPM) task group 224 published a report titled "Comprehensive Proton Therapy Machine Quality Assurance" in August 2019 [22]. It describes the various beam delivery techniques in PT and is recommended as a good reference in PT QA. It categorizes 4 parts: dosimetry QA, mechanical (gantry, couch, snout, MLC, and alignment between beam and Xray) QA, motion and radiation safety [30], and imaging QA. The medical physicist in a PT facility should determine their optimized machine QA period and check items, after considering machine specifications and clinical protocol.

\section{Treatment planning and DQA}

The range uncertainty of the treatment proton beam is the most sensitive and critical parameter on proton treatment planning. Especially, the patient who has any implanted metallic material on the beam pathway should be considered carefully because this changes the beam range and perturbs dose distribution on treatment planning [31,32]. TPS has a limitation; it must calculate dose distribution accurately in the inhomogeneous tissue region with analytical beam modeling, and the Monte Carlo simulation method has recently been adapted to improve the dose calculation accuracy. The variable RBE [33] consideration is also a recent emerging issue in plan optimization. Currently, the recommendation for proton RBE values is 1.1 in clinic. However, it increases more than 1.1 in the distal falloff and could damage the OARs near the tumor. As a future feature of TPS, the proton RBE models based on LET and the $\alpha / \beta$ parameter of the linear quadratic model should be introduced for plan optimization. The physicist should carefully quantify the effects of patient setup variation, intra- and inter-fraction motion, and range uncertainty based on the specifications of beam delivery, patient imaging, and the alignment system in their PT facility. Especially, the range uncertainty of the treatment beam depends on the pathway and tissue type in the pathway. So, the field specific margin of target and beam angle dedicated planning target volume (PTV) are needed when planning. Generally, the uncertainty of $\mathrm{HU}$ versus relative stopping power (RSP) is $2.5 \%$ to $2.7 \%$ of range and setup error is $1.0-3.0 \mathrm{~mm}$ in PT. For the evaluation of target coverage and dose to the OARs in treatment, worst case scenarios need to be simulated and calculated with the internal margins and safety margins before the decision on a treatment plan. This is called "robustness evaluation." To achieve a good robust treatment plan, close communication is necessary between the physicist and physician for potential target coverage limitations and possible OAR overdose. There are two proton beam delivery techniques: the passive beam mode using aperture and range compensator [34] and the PBS mode. The DQA of passive beam mode checks output factor, beam range, and 


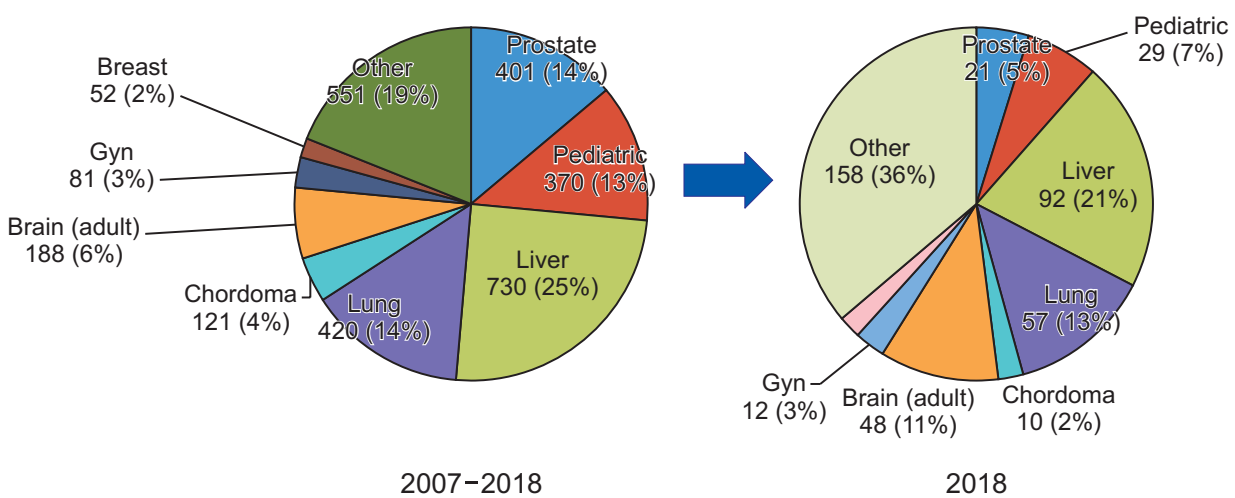

Fig. 12. The distribution of proton patient indications and changes of disease indications in 2018 at the National Cancer Center in Korea. width of SOBP [35]. The DQA of the PBS plan is similar to the X-ray IMRT DQA and evaluates the 2D dose distribution at several depths on the target volume.

\section{Future of PT}

\section{Extending indications for PT}

The total number of PT patients at NCC in Korea was about 3,300 at the end of 2019, and the daily number of patients is about 40. Fig. 12 shows the PT indications at the NCC in Korea. The major indication was prostate cancer cases before 2015 because PT was not reimbursed by National Health Insurance expect for pediatric cancer. Since the National Health Insurance started reimbursing for almost all cancers except for breast and prostate, liver, lung, and brain cancer patients have increased at the NCC in Korea. The ratio of proton treatment patients to total new patients in the department of radiation oncology doubled from $7 \%-8 \%$ to $15 \%-16 \%$.

\section{Compact size and cost effective system}

The most compact PT is the Mevion system among the currently running PT systems. Its footprint is less than 180 $\mathrm{m}^{2}$. Vendors are trying to develop PT solutions of more compact size with more user friendly operational systems. This will reduce the maintenance cost of PT systems and building construction costs. Another way to create a cost effective system is to increase patient throughput. Dynamic volumetric proton arc therapy can provide high patient throughput by reducing beam delivery time and patient setup time.

\section{More conformal PT}

Proton beam range uncertainty mainly comes from converting the CT-HU value to proton stopping power ration. At present, proton planners apply about $3.5 \%$ beam range margin. There are several efforts, like proton CT, multienergy X-ray CT, and Monte Carlo simulation method to reduce it.

\section{Is flash therapy the future of PT?}

Developing a technique for flash therapy is a hot topic for the big vendors of PT. There are many challenging issues before this can be a practical technique for clinical use, as I mentioned in the previous section. If it is successful, it will be a revolutionary technique to open a new era in PT. Because it will reduce total patient treatment time dramatically, with one day and less than 1 second beam irradiation time keeping clinical benefit. It can also enlarge the clinical indication, like a moving target. Still, there are unknown mechanisms and many technical barriers to overcome.

\section{Conclusions}

PT is the main modality in radiation therapy, and it plays a large part in cancer treatment. Thirty years in a hospitalbased PT facility has shown impressive clinical benefits and outcomes. Because of the development of compact facilities and user friendly operation, the number of proton facilities are rapidly increasing. Furthermore, there are po- 
tential techniques that can support new benefits in clinic. If the price of a PT solution is comparable to X-ray treatment solutions, I think the number of PT sites will increase more quickly, and it will be the solution of choice for many IMRT indication patients in radiation therapy. From a medical physicist aspect, $\mathrm{PT}$ is challenging because beam dosimetry and treatment planning are very different compared to the familiar X-ray therapeutic beam. Also, there are still many topics that could be developed for the matured solution.

\section{Acknowledgements}

I would like to appreciate IBA and Mevion that they provided their picture and technical information of their proton therapy solution. I also would like to thank people in proton therapy center, NCC for their valuable discussion for proton therapy system.

\section{Conflicts of Interest}

The author has nothing to disclose.

\section{Availability of Data and Materials}

All relevant data are within the paper and its Supporting Information files.

\section{References}

1. Leo WR: The bethe-bloch formula. Techniques for nuclear and particle physics experiments. 2nd ed. Berlin, Heidelberg: Spriger-Verlag; 1994.

2. Shin J, Park S, Kim H, Kim M, Jeong C, Cho S, et al. Proton linear energy transfer measurement using emulsion cloud chamber. Nucl Instrum Methods Phys Res B. 2015;349:201208.

3. International Commission on Radiation Units \& Measurements. Report 78: prescribing, recording, and reporting proton-beam therapy. Bethesda: International Commission on Radiation Units \& Measurements; 2007.

4. Baek HJ, Kim TH, Shin D, Kwak JW, Choo DW, Lee SB, et al. Radiobiological characterization of proton beam at the National Cancer Center in Korea. J Radiat Res. 2008;49:509-
515.

5. Lawrence E, Livingston $M$. The production of high speed protons without the use of high voltages. Phys Rev. 1931;38: 834.

6. Giap H, Giap B. Historical perspective and evolution of charged particle beam therapy. Transl Cancer Res. 2012;1:127-136.

7. Particle Therapy Co-Operative Group. Facilties in operation. Taipei: Particle Therapy Co-Operative Group, 2020 [cited 2020 May 13]. Available from: https://www.ptcog.ch.

8. Kim JY. Korea's first proton therapy center. Ilsan: Proton Therapy Today, 2012 [cited 2020 May 14]. Available from: http://www.proton-therapy-today.com/koreas-first-proton-therapy-center/.

9. Shin D, Yoon M, Kwak J, Shin J, Lee SB, Park SY, et al. Secondary neutron doses for several beam configurations for proton therapy. Int J Radiat Oncol Biol Phys. 2009;74:260265.

10. Jeong H, Rah JE, Hwang UJ, Yoo SH, Min BJ, Lee SY, et al. Estimation of the secondary cancer risk induced by diagnostic imaging radiation during proton therapy. J Radiol Prot. 2011;31:477-487.

11. Li X, Liu G, Janssens G, De Wilde O, Bossier V, Lerot X, et al. The first prototype of spot-scanning proton arc treatment delivery. Radiother Oncol. 2019;137:130-136.

12. Ding X, Li X, Zhang JM, Kabolizadeh P, Stevens C, Yan D. Spot-Scanning Proton Arc (SPArc) therapy: the first robust and delivery-efficient spot-scanning proton arc therapy. Int J Radiat Oncol Biol Phys. 2016;96:1107-1116.

13. Wilson JD, Hammond EM, Higgins GS, Petersson K. Ultrahigh dose rate (FLASH) radiotherapy: silver bullet or fool's gold? Front Oncol. 2020;9:1563.

14. Patriarca A, Fouillade C, Auger M, Martin F, Pouzoulet F, Nauraye C, et al. Experimental set-up for FLASH proton irradiation of small animals using a clinical system. Int J Radiat Oncol Biol Phys. 2018;102:619-626.

15. Beyreuther E, Brand M, Hans S, Hideghéty K, Karsch L, Leßmann E, et al. Feasibility of proton FLASH effect tested by zebrafish embryo irradiation. Radiother Oncol. 2019; 139:46-50.

16. Diffenderfer ES, Verginadis II, Kim MM, Shoniyozov K, Velalopoulou A, Goia D, et al. Design, implementation, and in vivo validation of a novel proton FLASH radiation 
therapy system. Int J Radiat Oncol Biol Phys. 2020;106:440448.

17. Paganetti H. Range uncertainties in proton therapy and the role of Monte Carlo simulations. Phys Med Biol. 2012; 57:R99-R117.

18. Handrack J, Tessonnier T, Chen W, Liebl J, Debus J, Bauer J, et al. Sensitivity of post treatment positron emission tomography/computed tomography to detect inter-fractional range variations in scanned ion beam therapy. Acta Oncol. 2017;56:1451-1458.

19. Kurz C, Bauer J, Unholtz D, Richter D, Herfarth K, Debus J, et al. Initial clinical evaluation of PET-based ion beam therapy monitoring under consideration of organ motion. Med Phys. 2016;43:975-982.

20. Min CH, Kim CH, Youn MY, Kim JW. Prompt gamma measurements for locating the dose falloff region in the proton therapy. Appl Phys Lett. 2006;89:183517.

21. Gensheimer MF, Yock TI, Liebsch NJ, Sharp GC, Paganetti $\mathrm{H}$, Madan $\mathrm{N}$, et al. In vivo proton beam range verification using spine MRI changes. Int J Radiat Oncol Biol Phys. 2010;78:268-275.

22. Arjomandy B, Taylor P, Ainsley C, Safai S, Sahoo N, Pankuch M, et al. AAPM task group 224: comprehensive proton therapy machine quality assurance. Med Phys. 2019;46: e678-e705.

23. Schneider U, Pemler P, Besserer J, Pedroni E, Lomax A, Kaser-Hotz B. Patient specific optimization of the relation between CT-hounsfield units and proton stopping power with proton radiography. Med Phys. 2005;32:195-199.

24. Jo K, Kim MY, Jeong JH, Jeang EH, Kim H, Park S, et al. A practical experience of dose modeling for proton pencil beam scanning in KNCC. J Korean Phys Soc. 2015;67:108115.

25. Kim DW, Lim YK, Shin J, Ahn S, Shin MY, Lee SB, et al. A dose verification method for proton therapy by using a plastic scintillation plate. J Korean Phys Soc. 2009;55:702708.
26. Cho S, Lee N, Song S, Son J, Kim H, Jeong JH, et al. Toward a novel dosimetry system using acrylic disk radiation sensor for proton pencil beam scanning. Med Phys. 2018;45:52775282.

27. Shin J, Kim D, Lim YK, Ahn S, Shin D, Yoon MG, et al. Monte Carlo modeling and simulation of a passive treatment proton beam delivery system using a modulation wheel. J Korean Phys Soc. 2010;56:153-163.

28. Kim DH, Kang YN, Suh TS, Shin J, Kim JW, Yoo SH, et al. Monte Carlo modeling and validation of a proton treatment nozzle by using the Geant4 toolkit. J Korean Phys Soc. 2012; 61:1125-1130.

29. Shin WG, Testa M, Kim HS, Jeong JH, Lee SB, Kim YJ, et al. Independent dose verification system with Monte Carlo simulations using TOPAS for passive scattering proton therapy at the National Cancer Center in Korea. Phys Med Biol. 2017;62:7598-7616.

30. Lee SH, Cho S, You SH, Shin D, Park SY, Lee SB, et al. Evaluation of radioactivity induced by patient-specific devices in proton therapy. J Korean Phys Soc. 2012;60:125-128.

31. Lim YK, Kwak J, Kim DW, Shin D, Yoon M, Park S, et al. Microscopic gold particle-based fiducial markers for proton therapy of prostate cancer. Int J Radiat Oncol Biol Phys. 2009;74:1609-1616.

32. Kwak J, Shin J, Kim JS, Park SY, Shin D, Yoon M, et al. Dosimetric influence of implanted gold markers in proton therapy for prostate cancer. Korean J Med Phys. 2010;21:291-297.

33. Sánchez-Parcerisa D, López-Aguirre M, Dolcet Llerena A, Udías JM. MultiRBE: Treatment planning for protons with selective radiobiological effectiveness. Med Phys. 2019;46: 4276-4284.

34. Park S, Jeong C, Kang DY, Shin JI, Cho S, Park JH, et al. Proton-radiography-based quality assurance of proton range compensator. Phys Med Biol. 2013;58:6511-6523.

35. Kim DW, Lim YK, Ahn SH, Shin J, Shin D, Yoon M, et al. Prediction of output factor, range, and spread-out Bragg peak for proton therapy. Med Dosim. 2011;36:145-152. 